\title{
Electronic properties of graphene nanoribbons doped with zinc, cadmium, mercury atoms
}

\author{
O. Omeroglu ${ }^{\mathrm{a}, *}$, E. Kutlu ${ }^{\mathrm{b}}$, P. Narin ${ }^{\mathrm{a}}$, S.B. Lisesivdin ${ }^{\mathrm{a}}$, E. Ozbay ${ }^{\mathrm{c}, \mathrm{d}, \mathrm{e}}$ \\ a Department of Physics, Faculty of Science, Gazi University, Teknikokullar, 06500 Ankara, Turkey \\ ${ }^{\mathbf{b}}$ Department of Energy Systems Engineering, Faculty of Engineering and Natural Sciences, Ankara Yuldrum Beyaztt University, 06010 Ankara, Turkey \\ ${ }^{\mathrm{c}}$ Nanotechnology Research Center, Bilkent University, Bilkent, 06800 Ankara, Turkey \\ d Department of Physics, Bilkent University, Bilkent, 06800 Ankara, Turkey \\ e Department of Electrical and Electronics Engineering, Bilkent University, Bilkent, 06800 Ankara, Turkey
}

\section{A R T I C L E I N F O}

\section{Keywords:}

Graphene nanoribbon

Doping

DFT

Zinc

Cadmium

Mercury

\begin{abstract}
A B S T R A C T
The effect of substitutional impurities as Zinc (Zn), Cadmium (Cd) and Mercury (Hg) on electronic properties of graphene nanoribbons (GNRs) was investigated by using Density Functional Theory (DFT). A substantial change in the electronic properties of GNR structures was observed while changing the position of dopant atom from the edge to the center of armchair graphene nanoribbons (AGNRs) and zigzag graphene nanoribbons (ZGNRs). The calculations are shown that the electronic band gap of GNRs can be controlled depending on the position of dopant atoms. The calculated electronic band structures for both AGNRs and ZGNRs show spin-dependent metallic or semiconductor behavior according to the position of dopant atoms. From the Density of States (DOS) information, quasi-zero-dimensional (Q0D) and quasi-one-dimensional (Q1D) type behaviors are observed. It is shown that because the doped ZGNRs had the lowest total energies, ZGNRs are energetically more stable than AGNRs.
\end{abstract}

\section{Introduction}

Graphene is two-dimensional material established by a sheet of carbon (C) atoms arranged in order a hexagonal lattice. It is an allotrope of carbon in the structure of a plane of $\mathrm{sp}^{2}$ bonded atoms with a molecule bond length of $0.142 \mathrm{~nm}$, has emerged as an interesting material of the 21st century [1]. Graphene and its different types have the potential to be used in various applications impact on electronic and optoelectronic devices, chemical sensors, nanocomposites and energy storage [2,3]. Graphene can be seen as the fundamental essential principle of a kind of materials with different dimensionalities, such as one-dimensional nanoribbons or nanotubes, zero-dimensional fullerenes or three-dimensional graphite [4-6]. The first of these, graphene nanoribbons are one-dimensional structures. ZGNR and AGNR are classified as zigzag and armchair according to shape of the edges in GNRs. It is known that in general ZGNRs are metallic, while AGNRs are known as the metallic or semiconductor properties depending on its widths and edge states [7-10]. AGNR is expressed as non-magnetic semiconductors while the magnetic ground states of ZGNR make them convenient a suitable for spintronic applications [11-15]. Theoretical and experimental investigations are still going on GNRs. In order to investigate these structures better, DFT has been applied to explore the electronic behavior. The determination of the electronic band structures of GNRs and the effects of the dopant atoms on the electronic band structures have been examined in various studies [16].

In the some theoretical studies that can be examined, transition metals (TMs) are used as a metal dopant or adatoms to achieve the spindependent structures $[17,18]$. It is already known that the electronic properties of GNRs are strongly dependent on the dopant atoms. With doping with some TMs, it has been reported significant changes in electronic band structures of the GNRs [19,20]. Correspondingly, using TMs it has provided the opportunity to investigate the changes in the spin dependencies and to control the electronic band gap.

Previous results obtained that the doping location along the nanoribbons are important in terms of manipulating the electronic properties of GNRs [21]. In this study, the effect of group IIB metals as Zn, $\mathrm{Cd}$ and $\mathrm{Hg}$ substitutional doping at four successive positions of the ZGNRs and the AGNRs are studied using DFT method with spin-polarized generalized gradient approximation (SGGA) as exchange-correlation. The electronic behavior of the doped GNRs has been explained in detail by calculating the band structures, the density of states (DOS), the total energies of the investigated structures.

\footnotetext{
* Corresponding author.

E-mail address: omerogluoznur@gmail.com (O. Omeroglu).
} 

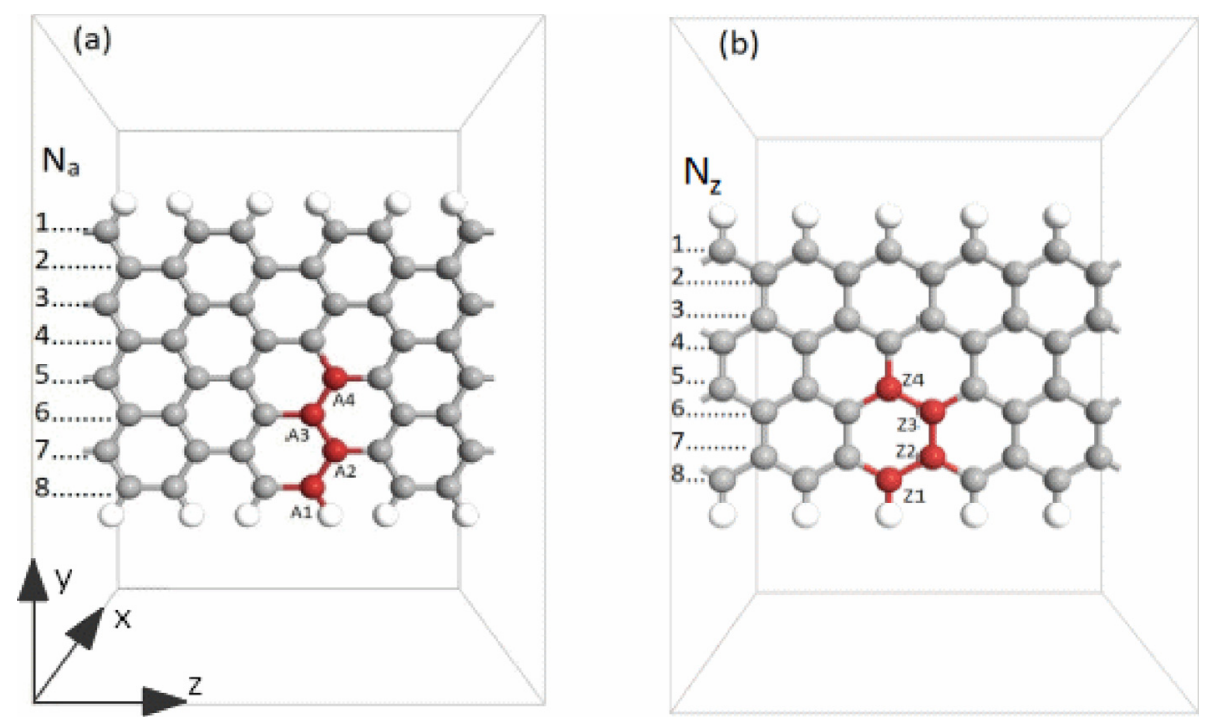

Fig. 1. The supercell of graphene nanoribbons with substitutional doping for (a) AGNR, (b) ZGNR.

\section{Computational details}

The calculations were based on spin-polarized DFT using SGGA, implemented in the Atomistix ToolKit-Virtual NanoLab (ATK-VNL) software for the investigated structures [22]. For the integration of the Brillouin zone, $1 \times 1 \times 100$ k-points were used within the MonkhorstPack scheme [23]. In the calculations, the AGNRs and ZGNRs passivated with hydrogen $(\mathrm{H})$ atoms for the different atom widths $\left(N_{a}\right.$ for AGNR and $N_{z}$ for ZGNR) have been investigated. Because nanoribbons with different widths are exhibited similar behavior to each other in the calculations, only nanoribbons had 8 atoms width have been investigated in detail in this study. The density mesh cut-off energy was used as $500 \mathrm{eV}$ and the maximum applied force to the nanoribbons was selected less than $0.05 \mathrm{eV} / \AA$. The investigated nanoribbons are constrained two directions along $\mathrm{x}$ and $\mathrm{y}$-axis, while they are infinite in the z-direction.

Supercells of AGNR and ZGNR had 8 atoms widths are shown in Fig. 1. Gray, white and red atoms are represented $\mathrm{C}, \mathrm{H}$ and substitutional dopant atoms ( $\mathrm{Zn}, \mathrm{Cd}, \mathrm{Hg}$ ), respectively. The electronic band structure and the DOS have been carried out for the A1, A2, A3, A4 doping sites of the AGNRs and Z1, Z2, Z3, Z4 doping sites of the ZGNRs. To determine the energetically stable structure, the total energy $\left(E_{T}\right)$ of each structure has been calculated.

\section{Results and discussion}

\subsection{Effect of group IIB atoms on electronic properties of AGNRs}

The electronic band structures of the AGNRs doped with $\mathrm{Zn}$ atom are shown for different dopant atom sites in Fig. 2. From now on, in the electronic band structure representations of the investigated structures, spin up and spin down bands are shown black and red lines, respectively. In Fig. 2(a), when the $\mathrm{Zn}$ doped structure at the A3 site has a band gap of $0.08 \mathrm{eV}$ for the spin down band and metallic behavior for the spin up band. Considering the electronic band structure for all dopant atom sites in Fig. 2(a), the calculated band gap results show that AGNRs doped with the $\mathrm{Zn}$ atom have metallic behavior. In the investigated AGNR structures, spin-dependent electronic band structure is observed. Fig. 2(b) shows the DOS of the structures doped with $\mathrm{Zn}$ atom from A1 to A4 sites. Non-equilibrium spin density in which one spin band shows conducting behavior while the other one state reveals semiconducting behavior. Generally, while the spin up bands are getting rarer, spin down bands are getting denser around the Fermi level. Also, Q0D behaviors have been observed at A2, A3, A4 sites in the DOS near the Fermi level [23].

The band structures of Cd doped AGNRs are shown in Fig. 3(a). Spin-dependent semiconductor behavior in the electronic band structures is shown in the investigated AGNRs with Cd dopant atom. These structures have direct band gaps with changing between $0.36 \mathrm{eV}$ and $0.46 \mathrm{eV}$. In Fig. 3(b), the DOS of Cd doped AGNR are shown. In doping at A3 site case, dense spin up band are found to be formed near the Fermi level.

The band structures of $\mathrm{Hg}$ doped AGNRs are shown in Fig. 4(a). For $\mathrm{Hg}$ doping at $\mathrm{A} 1$ and $\mathrm{A} 2$ sites, the electronic band gaps of the structures are calculated as $0.59 \mathrm{eV}$ and $0.23 \mathrm{eV}$ for the spin up bands, respectively. As Hg atom settled into the center of AGNR, the spin dependent properties of the band structure are disappeared as is seen from A3 and A4 sites. When the position of the dopant atom is changed, the energy levels of both spin up and spin down bands are started to overlap. The calculated results show that Zn doped AGNRs have a metallic behavior whereas $\mathrm{Cd}$ and $\mathrm{Hg}$ doped AGNRs are exhibited the semiconductor properties.

In Fig. 4(b), it is shown that $\mathrm{Hg}$ doped AGNR structures exhibit semiconductor behavior. As the position of the dopant atom shifts to the center of AGNR, the electronic states are getting further away from the Fermi level. For doped structures at A1, A2 and A3 sites, with settle like states near to the Fermi level, show typical Q0D behavior [24].

\subsection{Effect of group IIB atoms on electronic properties of ZGNR}

The electronic band structures of Zn doped ZGNRs are shown in Fig. 5(a). The calculations show that ZGNRs with doped Zn atom at Z1 and $\mathrm{Z} 4$ sites have metallic behavior. Also, the spin dependency in the electronic structure for the $\mathrm{Z} 2$ site with $0.50 \mathrm{eV}$ and $\mathrm{Z} 3$ site with $0.70 \mathrm{eV}$ band gap values of ZGNRs is observed as the semiconductor behavior for the spin down bands. As doped atom approaches the center of the 

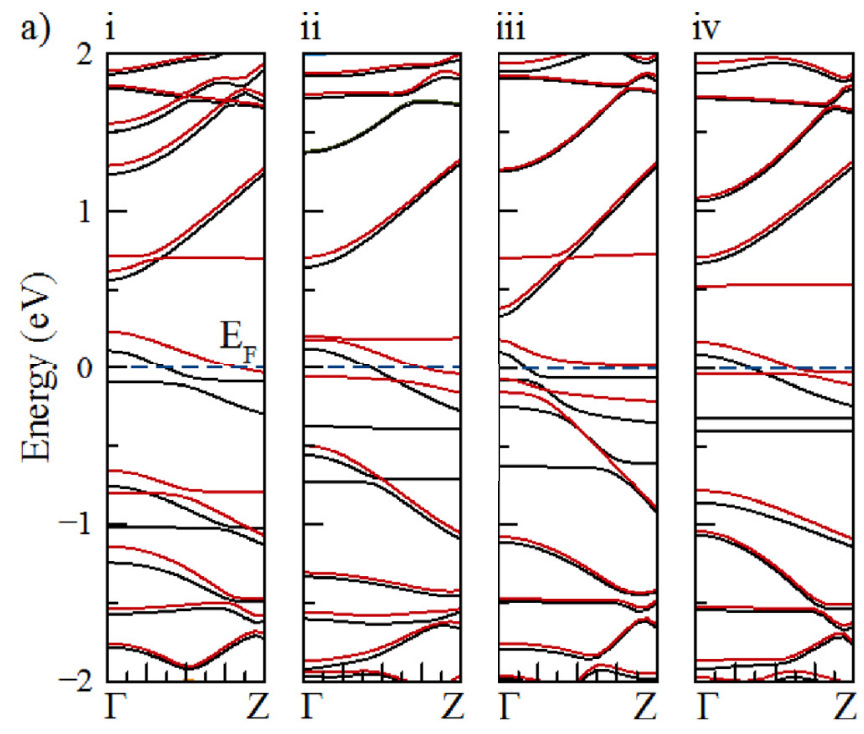

b)
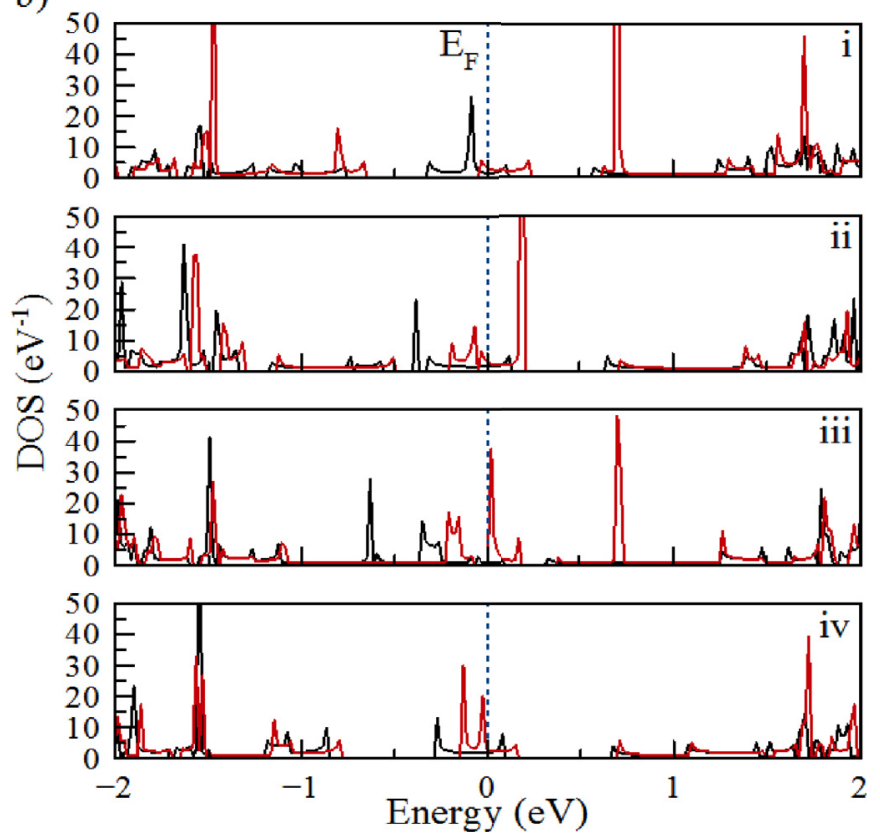

Fig. 2. a) Band structures and b) DOS of AGNRs doped with Zn atom for different doping sites, i) A1, ii) A2, iii) A3, iv) A4. Fermi level is shown with the blue dashed line in this study. (For interpretation of the references to colour in this figure legend, the reader is referred to the Web version of this article.)

structure, the band gap closes. In Fig. 5(b), the Z1 structure shows broad, settle like, spin up and down bands with Q0D behavior just at Fermi level.

The electronic band structure of Cd doped ZGNRs are investigated in Fig. 6(a). The band gaps of ZGNRs with doped Cd at Z1, Z2, and Z3 sites are calculated as $0.48 \mathrm{eV}, 0.13 \mathrm{eV}$ and $0.11 \mathrm{eV}$, respectively and exhibit semiconductor behavior with decreasing band gap values. For doping at center of the structure, namely the Z4 site, the electronic band structure shows metallic behavior. DOS results are shown in
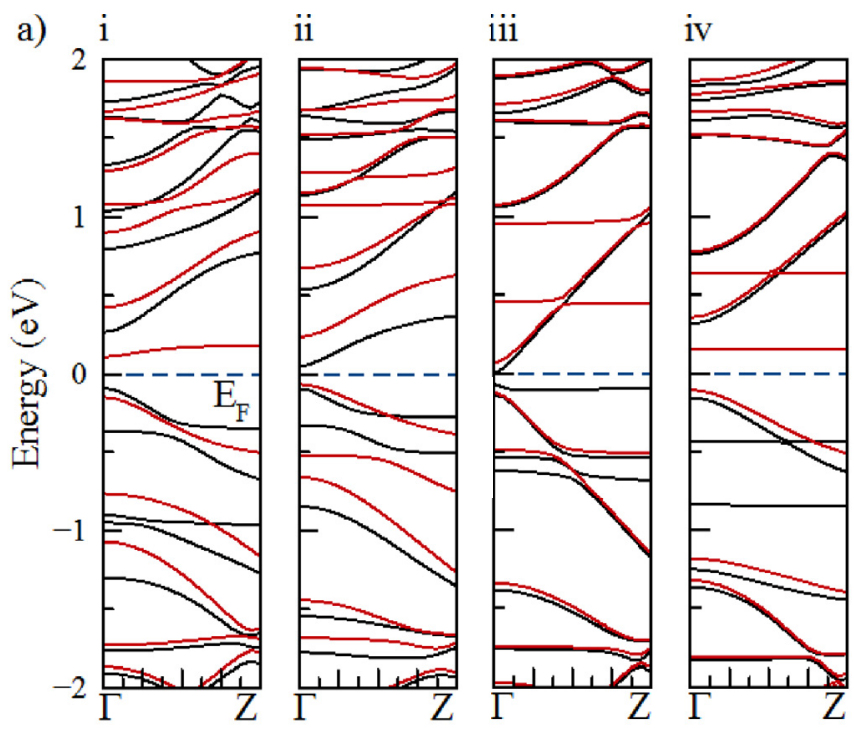

b)
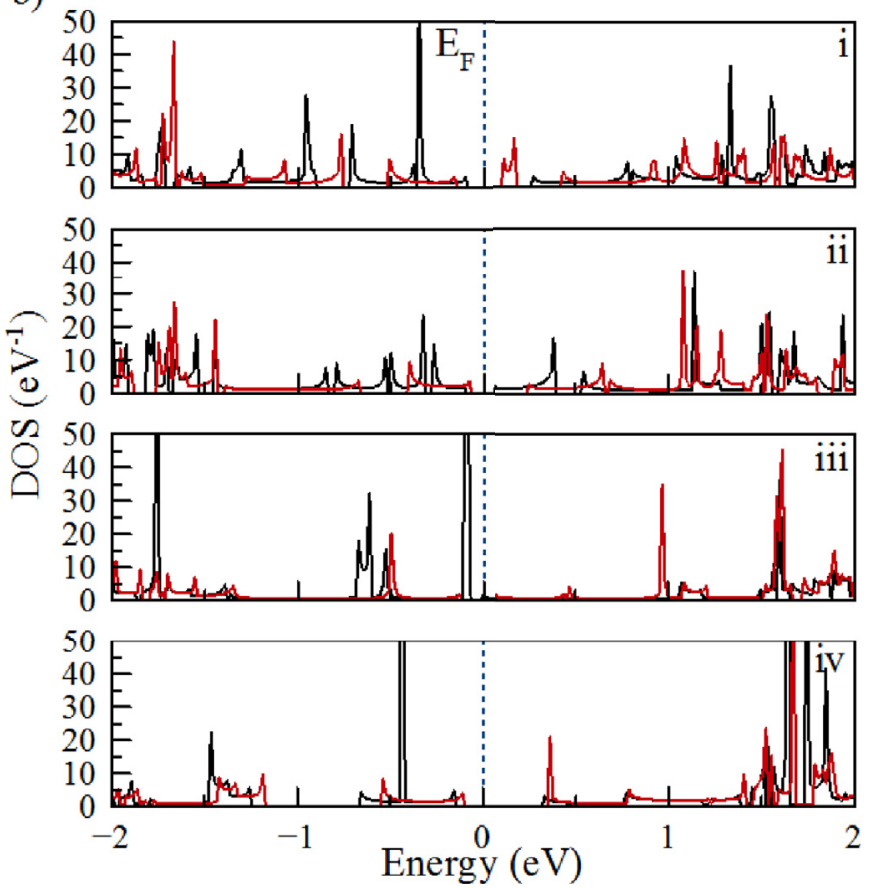

Fig. 3. a) Band structures and b) DOS of AGNRs doped with Cd atom for different doping sites, i) A1, ii) A2, iii) A3, iv) A4.

Fig. 6(b). It is shown that the electronic band gap is narrower as the dopant atom is moved to the center of ZGNR. Also, the Q0D and the Q1D behaviors have been observed in the DOS results [25,26].

The electronic band structure of $\mathrm{Hg}$ doped ZGNRs are shown in Fig. 7(a). As it is seen in $\mathrm{Hg}$ doped ZGNRs, the investigated structures for different doping sites exhibit semiconductor behavior except for Z4 site which shows the metallic behavior. In Fig. 7(b), DOS results clearly show that similar to the ZGNR with Cd, as dopant atom placed near to the center of the structure, the band gap of the structures is getting smaller. 

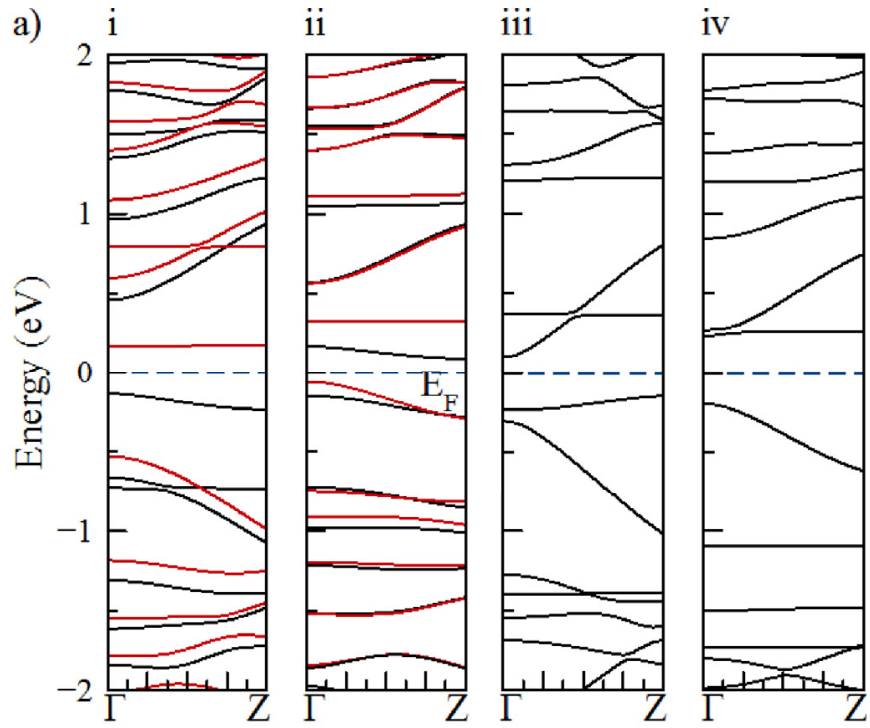

b)
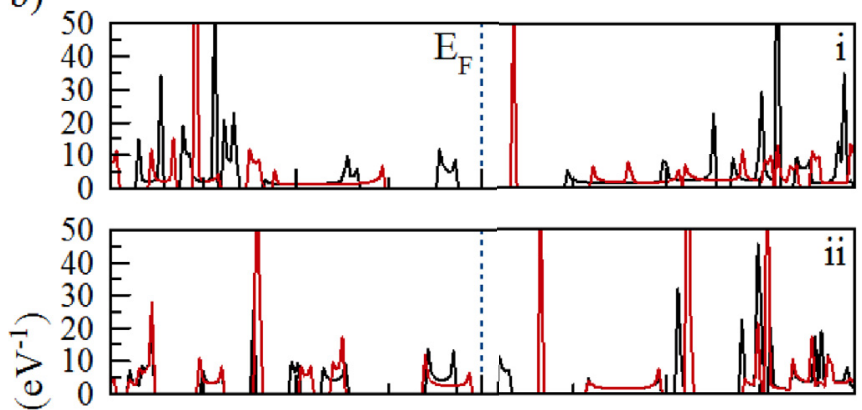

50
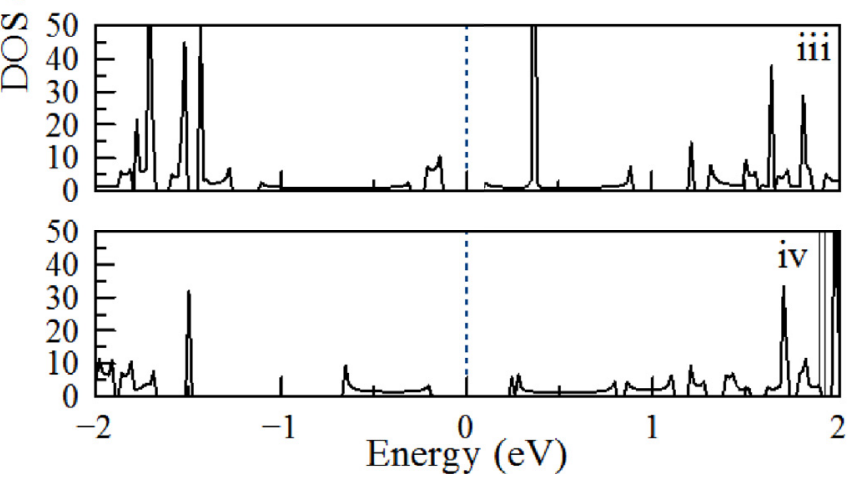

Fig. 4. a) Band structures and b) DOS of AGNRs doped with $\mathrm{Hg}$ for different doping sites, i) A1, ii) A2, iii) A3, iv) A4 are given.

Substitutional doping with $\mathrm{Zn}, \mathrm{Cd}$ and $\mathrm{Hg}$ dopant atoms have generated additional bands in the electronic band structure of the AGNRs and ZGNRs. Depending on the doping sites and type of the dopant atom, differences in the total energy values have been obtained. The total energies per $C$ atom and the energy band gap values of each investigated structure doped with group IIB atoms have been given in Table 1, Table 2 and Table 3. The bare AGNR and bare ZGNR for total energy per $\mathrm{C}$ atom were calculated as $-125.26 \mathrm{eV}$ and $-125.08 \mathrm{eV}$, respectively.

The ZGNRs doped with $\mathrm{Zn}$ atom reach the lowest total energies and
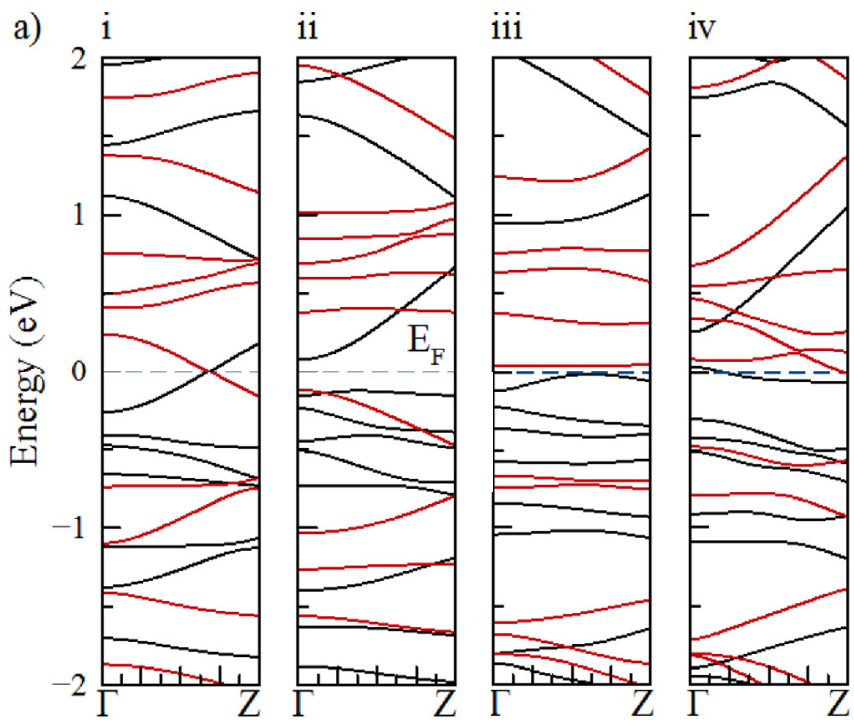

b)
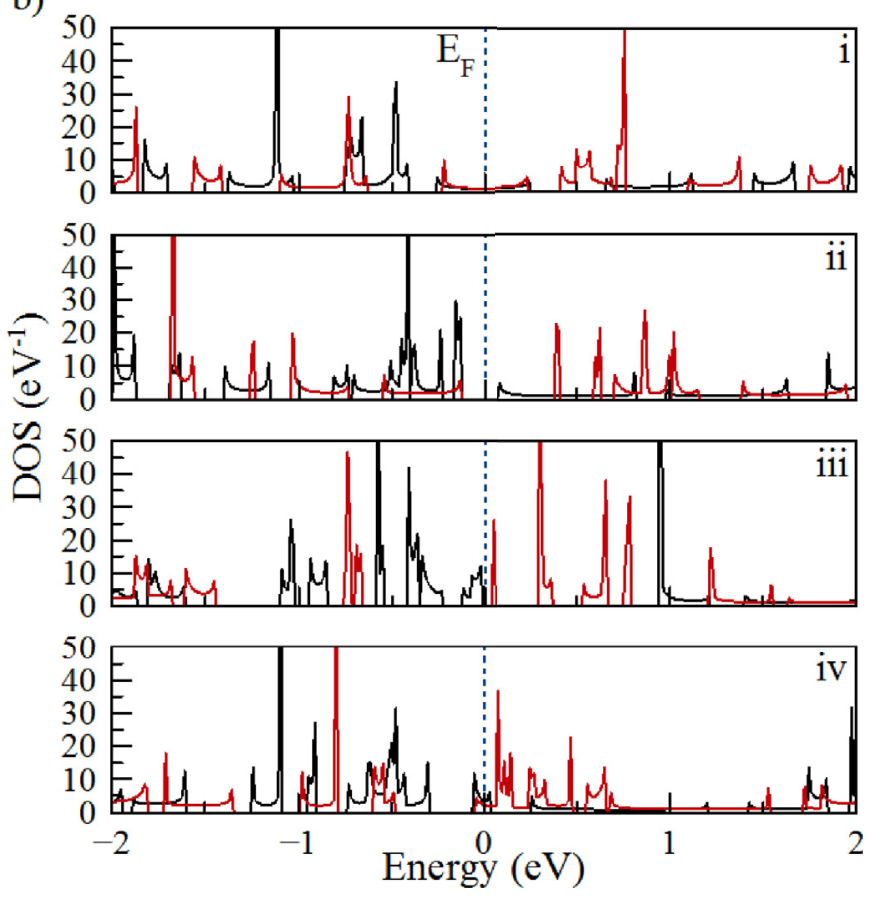

Fig. 5. a) Band structures and b) DOS of ZGNRs doped with Zn atom for different doping sites, i) Z1, ii) Z2, iii) Z3, iv) Z4.

more stable than other structures doped with $\mathrm{Cd}$ and $\mathrm{Hg}$ atoms. Generally, the total energies of doped ZGNRs are energetically more favorable than AGNRs. For GNRs doped with Zn, the structures with dopant near to the center of the ribbon are energetically more favorable than the structures with dopant near to the edge of the ribbon. However, for $\mathrm{Cd}$ and $\mathrm{Hg}$ doped structures an opposite behavior is calculated. For $\mathrm{Cd}$ and $\mathrm{Hg}$ doped structures with dopant near to the edge of the ribbon are energetically more favorable than structures with dopant near to the center of the ribbon. 

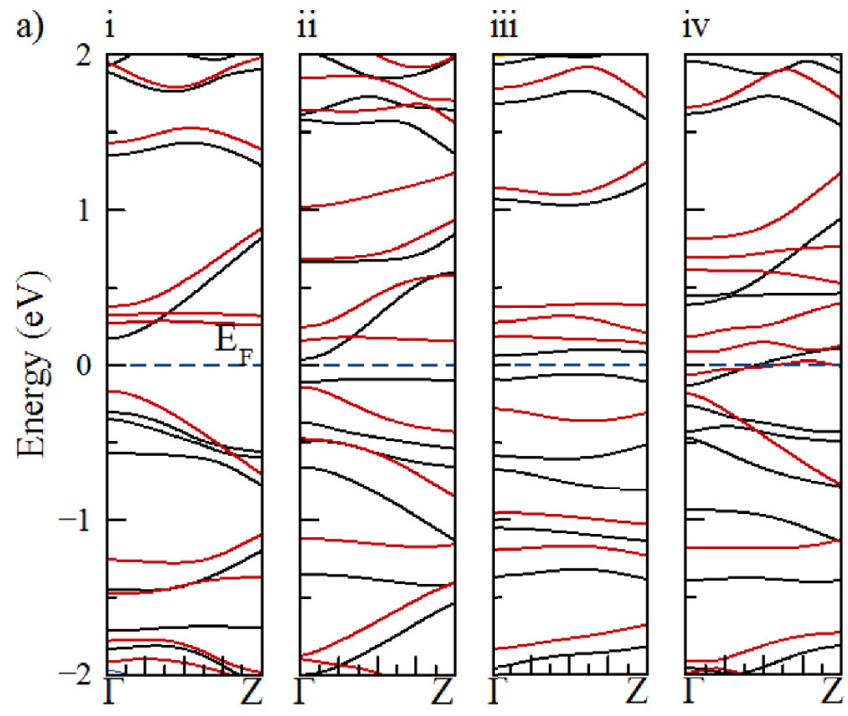

b)
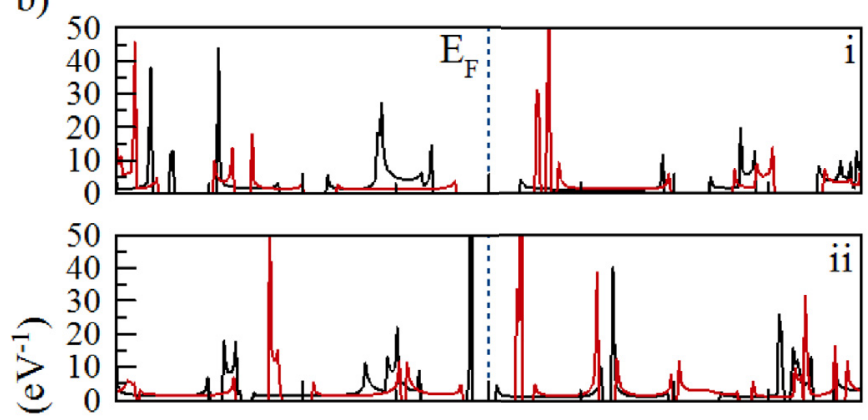

250
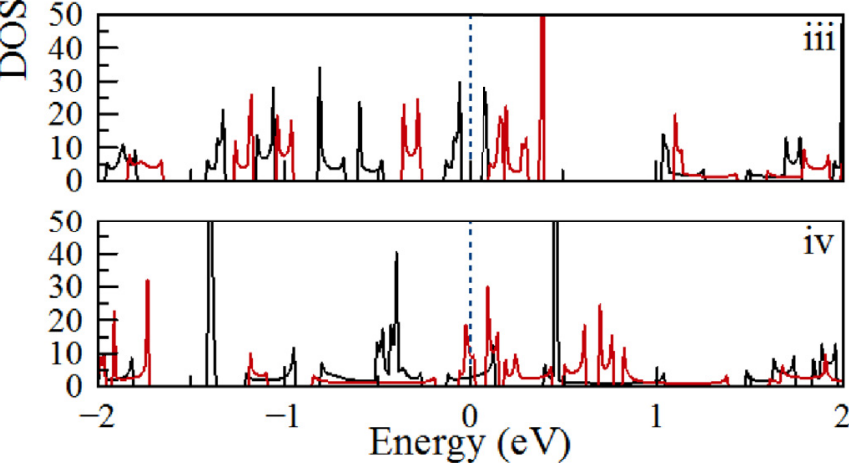

Fig. 6. a) Band structures and b) DOS of ZGNRs doped with Cd atom for different doping sites, i) Z1, ii) Z2, iii) Z3, iv) Z4.

\section{Conclusion}

The effect of dopant atoms on the electronic properties of GNRs has been studied by first-principles calculations using DFT. The AGNRs and ZGNRs with the substitutional dopant atoms provide a large scale of electronic properties based on different doping positions for the same ribbon width. Most of the investigated structures show magnetic properties with different doping sites except $\mathrm{Hg}$ atom. For $\mathrm{Hg}$ doped structures, the doping sites near the center of the ribbon do not produce spin dependent behavior. The electronic band structure for all of ZGNR
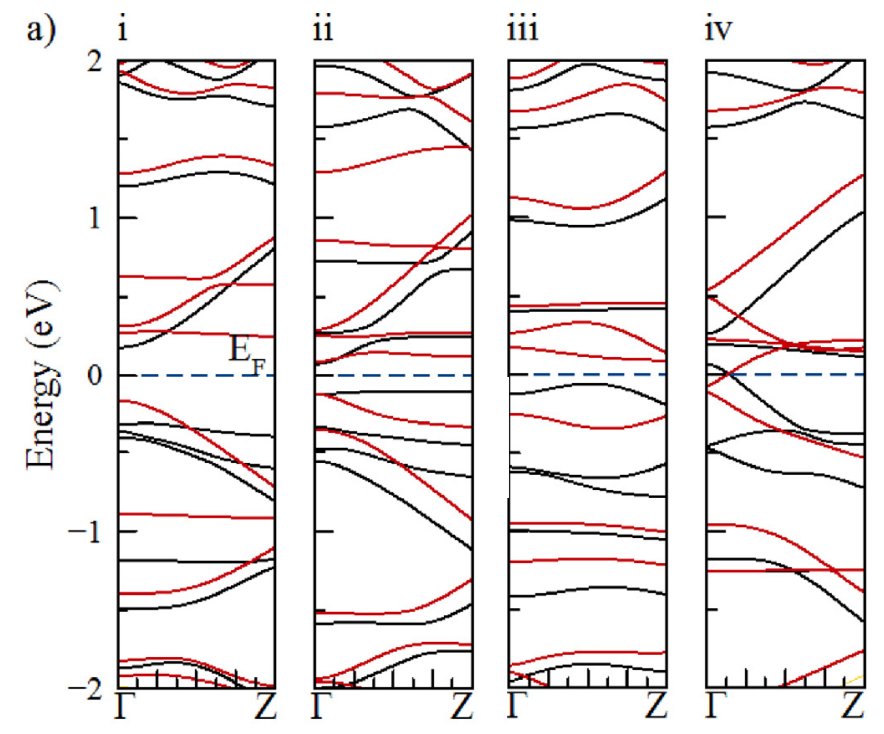

b)
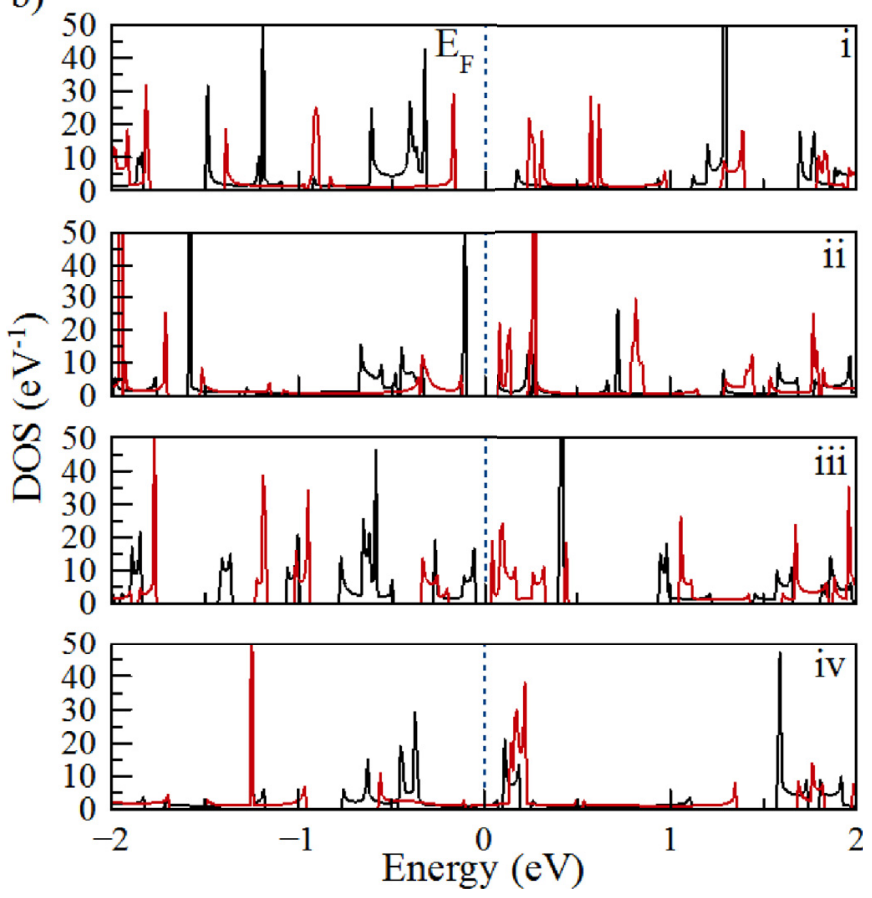

Fig. 7. a) Band structures and b) DOS of ZGNRs doped with $\mathrm{Hg}$ atom for different doping sites, i) Z1, ii) Z2, iii) Z3, iv) Z4.

reveals spin-dependent metallic behavior. Among the structures with different dopant atoms, the $\mathrm{Zn}$ doped GNRs is more stable than other structures. In the DOS calculations of many investigated cases, Q0D and Q1D type density behaviors have been found. Some of these behaviors of states which are near or at the Fermi level which directly affect the electronic behavior of the investigated structure. These results present the significance of changing the electronic properties of GNRs by controlling the placement of doping. 
Table 1

Total energies per C atom and energy band gap of AGNR and ZGNR structures doped with $\mathrm{Zn}$ atom for $N_{a, z}=8$. Structures without any band gap value shows metallic behavior.

\begin{tabular}{llllll}
\hline \multirow{2}{*}{ Site } & Total energy per C atom $(\mathrm{eV})$ & \multicolumn{2}{l}{ Spin up } & \multicolumn{3}{c}{ Spin down } \\
\cline { 3 - 6 } & & Eg (eV) & Direct & Eg (eV) & Direct \\
\hline A1 & -213.49 & - & - & - & - \\
A2 & -213.48 & - & - & - & - \\
A3 & -213.50 & - & - & 0.08 & No \\
A4 & -213.51 & - & - & - & - \\
Z1 & -229.69 & - & - & - & - \\
Z2 & -229.50 & 0.21 & No & 0.50 & No \\
Z3 & -229.76 & 0.96 & No & 0.70 & Yes \\
Z4 & -229.53 & - & - & - & - \\
\hline
\end{tabular}

Table 2

Total energies per C atom and energy band gap of AGNR and ZGNR structures doped with Cd atom for $N_{a, z}=8$. Structures without any band gap value shows metallic behavior.

\begin{tabular}{llllll}
\hline \multirow{2}{*}{ Site } & Total energy per C atom (eV) & \multicolumn{2}{l}{ Spin up } & \multicolumn{3}{c}{ Spin down } \\
\cline { 3 - 6 } & & Eg (eV) & Direct & Eg (eV) & Direct \\
\cline { 3 - 6 } & & 0.36 & Yes & 0.26 & Yes \\
A1 & -193.65 & 0.14 & Yes & 0.30 & Yes \\
A2 & -193.63 & 0.06 & Yes & 0.19 & Yes \\
A3 & -193.53 & 0.46 & Yes & 0.25 & Yes \\
A4 & -193.52 & 0.48 & Yes & 0.43 & No \\
Z1 & -205.83 & 0.13 & No & 0.30 & Yes \\
Z2 & -205.80 & 0.11 & No & 0.37 & Yes \\
Z3 & -205.72 & - & - & - & - \\
Z4 & -205.71 & & & & \\
\hline
\end{tabular}

Table 3

Total energies per C atom and energy band gap of AGNR and ZGNR structures doped with $\mathrm{Hg}$ atom for $N_{a, z}=8$. Structures without any band gap value shows metallic behavior.

\begin{tabular}{llllll}
\hline \multirow{2}{*}{ Site } & \multirow{2}{*}{ Total energy per C atom (eV) } & \multicolumn{2}{l}{ Spin up } & \multicolumn{3}{c}{ Spin down } \\
\cline { 3 - 6 } & & Eg (eV) & Direct & Eg (eV) & Direct \\
\hline A1 & -193.48 & 0.59 & Yes & 0.69 & Yes \\
A2 & -193.37 & 0.24 & No & 0.38 & Yes \\
A3 & -193.39 & 0.23 & No & 0.23 & No \\
A4 & -193.17 & 0.43 & Yes & 0.43 & Yes \\
Z1 & -205.75 & 0.50 & Yes & 0.41 & No \\
Z2 & -205.72 & 0.18 & No & 0.21 & Yes \\
Z3 & -205.52 & 0.46 & No & 0.23 & Yes \\
Z4 & -205.35 & - & - & - & - \\
\hline
\end{tabular}

\section{Acknowledgement}

This work was supported by TUBITAK under Project No. 116F197. E.O. acknowledges partial support from the Turkish Academy of Sciences. S.B.L. was supported in part by the Distinguished Young Scientist Award of Turkish Academy of Sciences (TUBA-GEBIP 2016).

\section{References}

[1] K.S. Novoselov, A.K. Geim, S.V. Morozov, D. Jiang, Y. Zhang, S.V. Dubonos, A.A. Firsov, Electric field effect in atomically thin carbon films, Science 306 (5696) (2004) 666-669.

[2] P.R. Wallace, Positron annihilation in solids and liquids, Solid State Phys. 10 (1960) $1-69$.

[3] I.W. Frank, et al., Mechanical properties of suspended graphene sheets, J. Vac. Sci. Technol. B: Microelectron. Nanometer Struct. Process. Measure. Phenom. 25 (6) (2007) 2558-2561.

[4] D. Liu, W. Zhao, S. Liu, Q. Cen, Q. Xue, Comparative tribological and corrosion resistance properties of epoxy composite coatings reinforced with functionalized fullerene C60 and graphene, Surf. Coating. Technol. 286 (2016) 354-364.

[5] D.R. Cooper, B. D'Anjou, N. Ghattamaneni, B. Harack, M. Hilke, A. Horth, N. Majlis, M. Massicotte, L. Vandsburger, E. Whiteway, V. Yu, Experimental review of graphene, ISRN Condens. Matter Phys. 2012 (2012).

[6] M. Bellec, U. Kuhl, G. Montambaux, F. Mortessagne, Manipulation of edge states in microwave artificial graphene, N. J. Phys. 16 (11) (2014) 113023.

[7] T.C. Li, S.P. Lu, Quantum conductance of graphene nanoribbons with edge defects, Phys. Rev. B 77 (8) (2008) 085408.

[8] K. Nakada, M. Fujita, G. Dresselhaus, M.S. Dresselhaus, Edge state in graphene ribbons: nanometer size effect and edge shape dependence, Phys. Rev. B 54 (24) (1996) 17954.

[9] M. Fujita, K. Wakabayashi, K. Nakada, K. Kusakabe, Peculiar localized state at zigzag graphite edge, J. Phys. Soc. Jpn. 65 (7) (1996) 1920-1923.

[10] Y. Niimi, T. Matsui, H. Kambara, K. Tagami, M. Tsukada, H. Fukuyama, Scanning tunneling microscopy and spectroscopy of the electronic local density of states of graphite surfaces near monoatomic step edges, Phys. Rev. B 73 (8) (2006) 085421.

[11] Y. Kobayashi, K.I. Fukui, T. Enoki, K. Kusakabe, Edge state on hydrogen-terminated graphite edges investigated by scanning tunneling microscopy, Phys. Rev. B 73 (12) (2006) 125415.

[12] Z. Kan, C. Nelson, M. Khatun, Quantum conductance of zigzag graphene oxide nanoribbons, J. Appl. Phys. 115 (15) (2014) 153704.

[13] L. Yang, C.H. Park, Y.W. Son, M.L. Cohen, S.G. Louie, Quasiparticle energies and band gaps in graphene nanoribbons, Phys. Rev. Lett. 99 (18) (2007) 186801.

[14] M. Ezawa, Peculiar width dependence of the electronic properties of carbon nanoribbons, Phys. Rev. B 73 (4) (2006) 045432.

[15] L. Pisani, J.A. Chan, B. Montanari, N.M. Harrison, Electronic structure and magnetic properties of graphitic ribbons, Phys. Rev. B 75 (6) (2007) 064418.

[16] Z. Wang, J. Xiao, X. Li, Effects of heteroatom (boron or nitrogen) substitutional doping on the electronic properties of graphene nanoribbons, Solid State Commun. 152 (2) (2012) 64-67.

[17] F.V. Kusmartsev, W.M. Wu, M.P. Pierpoint, K.C. Yung, Application of graphene within optoelectronic devices and transistors, Applied Spectroscopy and the Science of Nanomaterials, Springer, Singapore, 2015, pp. 191-221.

[18] O. Hod, V. Barone, G.E. Scuseria, Half-metallic graphene nanodots: a comprehensive first-principles theoretical study, Phys. Rev. B 77 (3) (2008) 035411.

[19] N.K. Jaiswal, P. Srivastava, Structural stability and electronic properties of Nidoped armchair graphene nanoribbons, Solid State Commun. 151 (20) (2011) 1490-1495.

[20] N.K. Jaiswal, P. Srivastava, First principles calculations of cobalt doped zigzag graphene nanoribbons, Solid State Commun. 152 (15) (2012) 1489-1492.

[21] Y. Dong, M.T. Gahl, C. Zhang, J. Lin, Computational study of precision nitrogen doping on graphene nanoribbon edges, Nanotechnology 28 (50) (2017) 505602.

[22] Version 2017 QuantumWise A/S. Available at http://www.quantumwise.com .

[23] H.J. Monkhorst, J.D. Pack, Special points for Brillouin-zone integrations, Phys. Rev, B 13 (12) (1976) 5188.

[24] H.S. Cho, P.R. Prucnal, Density of states of quasi-two-one, and-zero dimensional superlattices, J. Vac. Sci. Technol. B 7 (6) (1989) 1363-1367.

[25] B. Sarikavak-Lisesivdin, S.B. Lisesivdin, E. Ozbay, Ab initio study of Ru-terminated and Ru-doped armchair graphene nanoribbons, Mol. Phys. 110 (18) (2012) 2295-2300.

[26] A.F. Kuloglu, B. Sarikavak-Lisesivdin, S.B. Lisesivdin, E. Ozbay, First-principles calculations of Pd-terminated symmetrical armchair graphene nanoribbons, Comput. Mater. Sci. 68 (2013) 18-22. 